\title{
The ALPINE-ALMA [C II] survey
}

\section{Little to no evolution in the [C II]-SFR relation over the last $13 \mathrm{Gyr}$}

\author{
D. Schaerer ${ }^{1,2}$, M. Ginolfi ${ }^{1}$, M. Béthermin ${ }^{3}$, Y. Fudamoto ${ }^{1}$, P. A. Oesch ${ }^{1}$, O. Le Fèvre ${ }^{3}$, A. Faisst ${ }^{4}$, P. Capak ${ }^{4,5,6}$, \\ P. Cassata ${ }^{7,8}$, J. D. Silverman ${ }^{9,10}$, Lin Yan ${ }^{11}$, G. C. Jones ${ }^{12,13}$, R. Amorin ${ }^{14,15}$, S. Bardelli ${ }^{16}$, M. Boquien ${ }^{17}$, \\ A. Cimatti ${ }^{18,19}$, M. Dessauges-Zavadsky ${ }^{1}$, M. Giavalisco ${ }^{20}$, N. P. Hathi2 ${ }^{21}$, S. Fujimoto ${ }^{5,6}$, E. Ibar $^{22}$, A. Koekemoer ${ }^{21}$, \\ G. Lagache ${ }^{3}$, B. C. Lemaux ${ }^{23}$, F. Loiacono ${ }^{18,19}$, R. Maiolino ${ }^{12,13}$, D. Narayanan ${ }^{5,24,25}$, L. Morselli ${ }^{7,8}$,

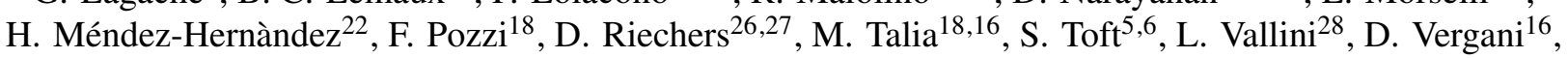 \\ G. Zamorani ${ }^{16}$, and E. Zucca ${ }^{16}$
}

(Affiliations can be found after the references)

Received 29 January 2020 / Accepted 29 April 2020

\begin{abstract}
The [C II] $158 \mu \mathrm{m}$ line is one of the strongest IR emission lines, which has been shown to trace the star formation rate (SFR) of galaxies in the nearby Universe, and up to $z \sim 2$. Whether this is also the case at higher redshift and in the early Universe remains debated. The ALPINE survey, which targeted 118 star-forming galaxies at $4.4<z<5.9$, provides a new opportunity to examine this question with the first statistical dataset. Using the ALPINE data and earlier measurements from the literature, we examine the relation between the [C II] luminosity and the SFR over the entire redshift range from $z \sim 4-8$. ALPINE galaxies, which are both detected in [C II] and in dust continuum, show good agreement with the local $L([C I I])-S F R$ relation. Galaxies undetected in the continuum by ALMA are found to be over-luminous in [C II] when the UV SFR is used. After accounting for dust-obscured star formation, by an amount of SFR(IR) $\approx \mathrm{SFR}(\mathrm{UV})$ on average, which results from two different stacking methods and SED fitting, the ALPINE galaxies show an $L([\mathrm{CII}])-$ SFR relation comparable to the local one. When [C II] non-detections are taken into account, the slope may be marginally steeper at high- $z$, although this is still somewhat uncertain. When compared homogeneously, the $z>6$ [C II] measurements (detections and upper limits) do not behave very differently to the $z \sim 4-6$ data. We find a weak dependence of $L([C I I]) / S F R$ on the $\operatorname{Ly} \alpha$ equivalent width. Finally, we find that the ratio $L([\mathrm{CII}]) / L_{\mathrm{IR}} \sim(1-3) \times 10^{-3}$ for the ALPINE sources, comparable to that of "normal" galaxies at lower redshift. Our analysis, which includes the largest sample ( $\sim 150$ galaxies $)$ of [C II] measurements at $z>4$ available so far, suggests no or little evolution of the [C II]-SFR relation over the last $13 \mathrm{Gyr}$ of cosmic time.
\end{abstract}

Key words. galaxies: high-redshift - galaxies: star formation - galaxies: formation

\section{Introduction}

The [C II] $158 \mu \mathrm{m}$ line is an important coolant of the neutral interstellar medium (ISM), one of the strongest emission lines in the infrared (IR), which is also emitted relatively close to the peak of dust continuum emission. Although [C II] has long been known to originate from H II regions, diffuse neutral and ionised ISM, and from photodissociation regions (e.g. Wolfire et al. 1995; Hollenbach \& Tielens 1999), it has been found to trace star formation. In particular, the [C II] luminosity has been shown to correlate well with the total star formation rate (SFR) of galaxies in our Galaxy, nearby galaxies, and up to $z \sim 2$ (see e.g. Pineda et al. 2014; Herrera-Camus et al. 2015; De Looze et al. 2011, 2014, and references therein). Other studies have recently stressed that [C II] could alternatively be used as a tracer of molecular gas (see e.g. Nordon \& Sternberg 2016; Glover \& Smith 2016; Fahrion et al. 2017; Zanella et al. 2018).

Since [C II] $158 \mu \mathrm{m}$ can be observed from the cosmic noon $(z \sim 2)$ out to very high redshift $(z \sim 7-8$, Inoue et al. 2016) with ALMA, and potentially even into the cosmic dark ages with other facilities (cf. Carilli et al. 2017), this line has often been targeted. The goal is to use it as a probe of the ISM properties in distant galaxies, as a measure of the total SFR (unaffected by the possible presence of dust), and for other purposes, including redshift confirmation for galaxies in the epoch of reionisation.
The first attempts to measure [C II] $158 \mu \mathrm{m}$ in galaxies at $z>6$ with ALMA have mostly been unsuccessful, essentially yielding non-detections, both for Ly $\alpha$ emitters (LAEs) and Lyman-break galaxies (LBGs; e.g. Ouchi et al. 2013; Ota et al. 2014; Maiolino et al. 2015). Subsequent observations have detected [C II] in LAEs and LBGs, both in blank fields and behind lensing clusters, finding several [C II]-underluminous galaxies at high- $z$ and suggesting a large scatter in $L$ ([CII])-SFR (see e.g. Maiolino et al. 2015; Willott et al. 2015; Pentericci et al. 2016; Bradač et al. 2017; Carniani et al. 2018) compared to the local samples (De Looze et al. 2014). On the other hand, Riechers et al. (2014) and Capak et al. (2015) successfully detected several $z \sim 5-6$ star-forming galaxies, revealing relatively broad [C II] lines and a good agreement with the local [C II]-SFR relation. Reanalysing the existing [C II] detections and non-detections of $z \sim 6-7$ galaxies, Matthee et al. (2019) showed that the available data appears compatible with the De Looze et al. (2014) relation for SFR $\gtrsim 30 M_{\odot} \mathrm{yr}^{-1}$ and may deviate from that of lower SFRs, if broader [C II] lines are assumed for the non-detections and the data are consistently compared. Conversely, using very similar data, Harikane et al. $(2018,2020)$ concluded that $z=5-9$ galaxies show a clear [C II] deficit with respect to the local [C II]-SFR relation, and that this deficit increases with increasing Ly $\alpha$ equivalent width. Manifestly, no consensus has yet been reached on these questions, and it is unclear if the [C II] $158 \mu \mathrm{m}$ line remains a good tracer 
of star formation at $z>4$ or if there is a quantitative change compared to the observations at low redshift.

To make progress on these issues, we used the ALMA Large Program to INvestigate $\mathrm{C}^{+}$at Early Times (ALPINE) survey, which targets 118 "normal" (i.e. main sequence) star-forming galaxies with known spectroscopic redshifts at $4.4<z<5.9$, and which is designed to provide the first statistical dataset making it possible to determine the observational properties of [C II] emission at high- $z$. The survey was recently completed and is described in detail in Le Fèvre et al. (2020), Bethermin et al. (2020), and Faisst et al. (2020). Our measurements, yielding 75 high-significance detections of [C II] $158 \mu \mathrm{m}$ and 43 nondetections, combined with the earlier [C II] observations of 36 galaxies at $z \sim 6-9.11$ from literature compilations, allow us to examine what is normal for high- $z$ galaxies and shed new light on the above questions.

The paper is structured as follows. We briefly summarise the ALPINE [C II] dataset and other measurements in Sect. 2. We then examine the behaviour of the [C II] $158 \mu \mathrm{m}$ luminosity with different SFR indicators, and we carefully compare the [C II]-SFR observations of high- $z$ galaxies to the reference sample of De Looze et al. (2014) (Sect. 3). We combine the ALPINE dataset with the available [C II] observations at $z>6$ and examine whether all high-redshift observations show the same picture and if the [C II]-SFR relation is different in the early Universe (Sect. 3.3). Finally, we present the observed [C II]-to-IR ratio in Sect. 4. We discuss the possible caveats and future improvements in Sect. 5. Our main results are summarised in Sect. 6, and we provide results to fits to different datasets in the appendix. We assume a $\Lambda \mathrm{CDM}$ cosmology with $\Omega_{\Lambda}=0.7, \Omega_{\mathrm{m}}=0.3$ and $H_{0}=70 \mathrm{~km} \mathrm{~s}^{-1} \mathrm{Mpc}^{-1}$, and a Chabrier IMF (Chabrier 2003).

\section{Observations and derived quantities}

The ALMA Large Program to INvestigate [C II] at Early times (ALPINE) survey, presented in Le Fèvre et al. (2020), has observed 118 "normal" star-forming galaxies with known spectroscopic redshifts at $4.4<z<5.9$. The ALPINE sample also includes seven galaxies (HZ1, HZ2, HZ3, HZ4, HZ5, HZ6/LBG1, and HZ8) that were previously observed with ALMA by Riechers et al. (2014) and Capak et al. (2015). It currently constitues the largest sample of [C II] observations at $z \sim 4-6$.

Details of the ALPINE data reduction and statistical source properties are described in Bethermin et al. (2020), from which we used the [C II $] 158 \mu \mathrm{m}$ line luminosities ( $L([\mathrm{C}$ II $]$ ), 75 detections with high significance, and 43 non-detections) and the dust continuum measurements ( 23 detections and 95 non-detections). The $158 \mu \mathrm{m}$ rest-frame continuum fluxes have been converted to total IR luminosities, $L_{\mathrm{IR}}$, using an average empirically based conversion from the $158 \mu \mathrm{m}$ monochromatic continuum flux density to $L_{\mathrm{IR}}$ as described in Bethermin et al. (2020). The empirical template gives a conversion similar to a modified black body with a dust temperature of $T_{\mathrm{d}}=42 \mathrm{~K}$, a dust opacity at $850 \mu \mathrm{m}$ of $k_{850}=0.077 \mathrm{~m}^{2} \mathrm{~kg}^{-1}$, and a grey-body power-law exponent $\beta=1.5$ (see e.g. Ota et al. 2014; Matthee et al. 2019).

For galaxies undetected in [C II], we used the "aggressive" $3 \sigma$ upper limits of $L([\mathrm{CII}])$ reported in Bethermin et al. (2020), defined as three times the RMS of the noise in velocityintegrated flux maps obtained by collapsing a channel width of $300 \mathrm{~km} \mathrm{~s}^{-1}$ centered around the expected spectroscopic redshift. We then rescaled these limits to reflect a more realistic (though less conservative) distribution of full width half maximum (FWHM) of our [C II]-undetected galaxies: motivated by the observed dependence of FWHM on $L([\mathrm{CII}])$ shown in

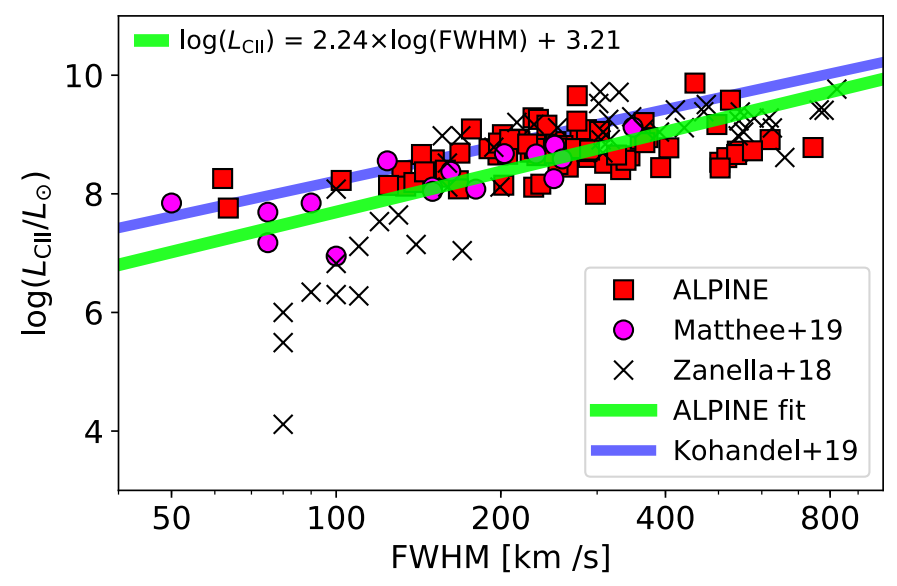

Fig. 1. Observed FWHM of the [C II] $158 \mu \mathrm{m}$ line as function of the [C II] luminosity from ALPINE and other data from the literature (the compilation of $z>6$ sources from Matthee et al. 2019) and available measurements for galaxies in the Zanella et al. (2018) compilation $(z \sim 0-6)$. The violet dashed line is a Tully-Fisher-like relation derived for high- $z$ galaxies by Kohandel et al. (2019). The green solid line shows our best fit to the data.

Fig. $1^{1}$, we adopted $F W H M=150 \mathrm{~km} \mathrm{~s}^{-1}$, instead of the median of $252 \mathrm{~km} \mathrm{~s}^{-1}$ of the [C II]-detected ALPINE galaxies (Bethermin et al. 2020). As discussed in Bethermin et al. (2020), we note that by construction our $3 \sigma$ upper limits of $L([\mathrm{CII}])$ can be underestimated if the sources are: (i) just below the detection threshold; (ii) spatially extended (larger than the beam-size, e.g., $\gtrsim 1^{\prime \prime}$ ); and/or (iii) show very broad line profiles (see e.g. Kohandel et al. 2019), where the two latter conditions are less likely to occur in less massive and less star-forming objects.

For galaxies undetected in continuum, we used the aggressive upper limits determined by Bethermin et al. (2020), using the same conversion from $158 \mu \mathrm{m}$ rest-frame continuum fluxes to total IR luminosity. Finally, we also used $L_{\mathrm{IR}}$ values derived from the IRX $-\beta$ relation obtained by stacking of the ALPINE sources, as described in Fudamoto et al. (2020). In the absence of a direct detection of dust continuum emission, this is our preferred method to correct for dust-obscured star formation.

From the rich dataset of ancillary photometric and spectroscopic data, which is also available for the ALPINE sources (see Faisst et al. 2020 for details), we used the observed UV luminosity (or equivalently the absolute UV magnitude $M_{1500}$ at $1500 \AA$ ). To compare our [C II] data with other measurements and results in the literature, we also used measurements of the Ly $\alpha$ equivalent widths, $\operatorname{EW}(\operatorname{Ly} \alpha)$, obtained from the rest-UV spectra of our sources, which were obtained during earlier spectroscopic observations with DEIMOS and VIMOS on the Keck and VLT telescopes. The spectra are discussed by Faisst et al. (2020). The Ly $\alpha$ measurements, available for 98 sources, are taken from Cassata et al. (2020), where a more detailed description of the Ly $\alpha$ properties is presented.

From the above-mentioned measurements of the UV and IR luminosities, we derived three "classical" measures of starformation rate, SFR(UV) uncorrected for attenuation, SFR(IR), and the total SFR(tot) = SFR(UV)+SFR(IR). We also used estimates of the total SFR, SFR(SED), obtained from the multi-band SED fits of Faisst et al. (2020). The different SFR measurements

\footnotetext{
1 A linear fit to the data yields $\log \left(L([\mathrm{CII}]) / L_{\odot}\right)=2.24 \times$ $\log \left(F W H M /\left(\mathrm{km} \mathrm{s}^{-1}\right)\right)+3.21$.
} 
are all included in the ALPINE database ${ }^{2}$, where the data will be made public.

To allow a proper comparison of the $L([\mathrm{CII}])-$ SFR relation with the low- $z$ galaxy sample of De Looze et al. (2014), we adopted the same conversion factors between $L_{\mathrm{UV}}, L_{\mathrm{IR}}$, and SFR as the ones used in their paper. We note that the SFR(UV) calibration adopted by De Looze et al. (2014) agrees with the classical one from Kennicutt (1998), when rescaled to the same IMF. However, for the same IMF, their IR calibration, taken from Murphy et al. (2011), yields 30\% (0.12 dex) higher SFR(IR) values than the Kennicutt (1998) calibration. Finally, we rescaled the SFR(UV) and SFR(IR) values by a factor of 1.06 from the Kroupa IMF (used by De Looze et al. 2014) to the Chabrier IMF, for consistency with the other ALPINE papers ${ }^{3}$. We highlight that we assume SFR(IR) $=0$ per default and unless otherwise stated for sources that are not detected in the continuum. This is discussed further below.

\section{Relations between the [ $\mathrm{C}_{\mathrm{II}} 158 \mu \mathrm{m}$ luminosity and SFR indicators at $z \sim 4-6$ and higher redshift}

\subsection{Comparing $L([C I I])$ with UV, IR, and SED-fit-based SFRs}

As is often done for high- $z$ galaxies, which are generally selected from the rest-UV and seldomly detected in the dust continuum, we first used a basic SFR indicator, SFR(UV) derived from the observed UV luminosity, which is available for the entire sample, to obtain the $L([\mathrm{CII}])-$ SFR relation shown in Fig. 2. The ALPINE data is compared to average relation of the low- $z$ H II -galaxy/starburst sample from De Looze et al. (2014) as a reference (henceforth named the "local" relation), which is often used in the literature. It includes 184 galaxies, shows a linear scaling between $L([\mathrm{CII}])$ and SFR, and a scatter of 0.27 dex (see their Table 3$)^{4}$. While the $[\mathrm{C}$ II $]$ detections span a wide range between $L([\mathrm{CII}]) \sim 5 \times 10^{7} L_{\odot}$ and $5 \times 10^{9} L_{\odot}$, SFR(UV) varies less, thus resulting in a relatively steep relation between $L([\mathrm{CII}])$ and SFR(UV). Compared to the local $L([\mathrm{CII}])-$ SFR correlation, the [C II] luminosity of our sources appears higher, in contrast to several high- $z(z \gtrsim 6)$ galaxies where [C II] was found to be " "under-luminous", as mentioned in the introduction. It is more likely that the SFR is underestimated, as can be expected from dust attenuation of the UV light.

To correct for dust attenuation in the simplest way, in the same figure (Fig. 2) we plot the [C II] measurements as a function of the total SFR, adding the dust-attenuated SFR(IR) to SFR(UV) for the galaxies for which we detect emission from the dust continuum. Clearly, for the continuum-detected sources, the increase in SFR is significant, bringing them to fair agreement with the local $L([\mathrm{CII}])-$ SFR relation, as seen by the comparison with the left panel. This corresponds to galaxies with $\mathrm{SFR}($ tot $) \gtrsim 30 M_{\odot} \mathrm{yr}^{-1}$.

On average, however, the [C II] luminosities of the 74 detected sources remain larger than expected from the local relation of De Looze et al. (2014), by a factor $\sim 1.5$ for the entire sample and a factor $\sim 2$ for the sources that are not

\footnotetext{
2 https://cesam.lam.fr/a2c2s/

3 In short, the final adopted SFR calibrations are: SFR(UV)/ $\left(M_{\odot} \mathrm{yr}^{-1}\right)=8.24 \times 10^{-29} L_{v}$, where $L_{v}$ is in units of ergs s${ }^{-1} \mathrm{~Hz}^{-1}$, or equivalently $\operatorname{SFR}(\mathrm{UV}) /\left(M_{\odot} \mathrm{yr}^{-1}\right)=1.59 \times 10^{-10} L_{\mathrm{UV}} / L_{\odot}$, where $L_{\mathrm{UV}}$ is calculated at $1500 \AA$. And SFR $(\mathrm{IR}) /\left(M_{\odot} \mathrm{yr}^{-1}\right)=1.40 \times 10^{-10} L_{\mathrm{IR}} / L_{\odot}$.

4 For comparison, their entire sample of 530 galaxies shows a larger scatter $(0.42 \mathrm{dex})$ and a [C II] $158 \mu \mathrm{m}$ luminosity, which is lower by 0.07 dex for a given SFR.
}

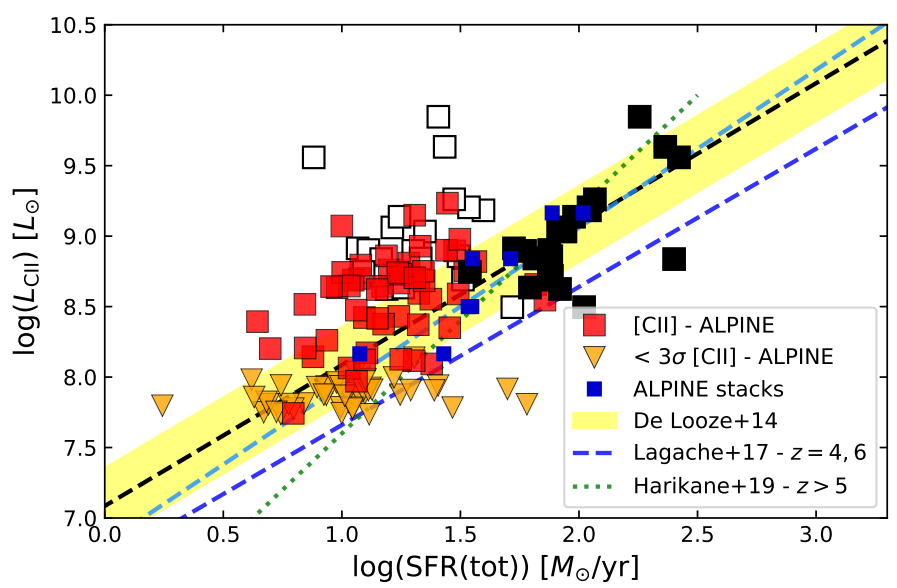

Fig. 2. [C II] as a function of UV or UV+IR-derived SFR for the $z \sim 4.5$ ALPINE sources. Squares show the $[\mathrm{C}$ II] detections, orange triangles the $3 \sigma$ upper limits. Black squares show galaxies with continuum detection (black filled squares show SFR(UV)+SFR(IR), empty squares the SFR(UV) of the same sources); red squares shows the SFR(UV) for the other (non-continuum-detected) ALPINE sources. Blue circles show the results from stacks of ALPINE sources in four bins of $L([\mathrm{C} \mathrm{II}])$ and two redshift bins, adapted from from Bethermin et al. (2020). The observations are compared to the [C II]-SFR relations of local galaxies determined by De Looze et al. (2014) adjusted to the Chabrier IMF by reducing the SFR by a factor of 1.06 (black dashed line), shown by the yellow band with a total width corresponding to $2 \sigma$. The green dotted line shows the relation fitted to observations of $z \sim 5-9$ galaxies by Harikane et al. (2020). The fits from the models of Lagache et al. (2018) for redshifts spanning the range of the observations are shown by the two blue dashed lines.

detected in the continuum (red squares in Fig. 2). Approximately $40 \%$ of the $[\mathrm{C}$ II $]$-detected ALPINE galaxies are extended and classified as mergers from a morphological and kinematic analysis (Le Fèvre et al. 2020). Excluding, for example, these mergers from the sample does not significantly change the deviation from the relation; on average, a shift by a factor 1.25 in SFR(tot) remains, compared to a factor 1.5 shift for the entire sample. For the mergers alone, the deviation is $0.28 \mathrm{dex}$, similar to that of several sources not detected in the continuum. From this, we conclude that even if there were systematic differences between mergers and galaxies in the local sample, this would probably not explain the observed deviation between the ALPINE dataset and the De Looze et al. (2014) relation. Obscured star formation, below our current detection threshold in the ALMA measurements, is probably present in the majority of the ALPINE sample.

Bethermin et al. (2020) carried out stacking of the ALPINE sources in different bins of [C II] luminosity, detecting thus the dust continuum in several of these bins, and hence measuring in particular the average dust-attenuated contribution SFR(IR). After conversion to the same SFR calibrations used here (cf. above) their results are shown in Fig. 2. The ALPINE stacking results show a good agreement with the local [C II]-SFR relation, indicating that some correction for dust-obscured star formation is necessary even for the continuum undetected galaxies, and especially those at the low $L([\mathrm{CII}])$ range.

Regrettably, the upper limits on the IR continuum fluxes of the individual ALPINE sources are not sufficiently constraining. Even if we use the aggressive $1 \sigma$ limits to determine a limit on hidden star formation by summing SFR(UV) and the SFR(IR) limit, we obtain the SFR(tot) limits shown in Fig. 3 (left), which 

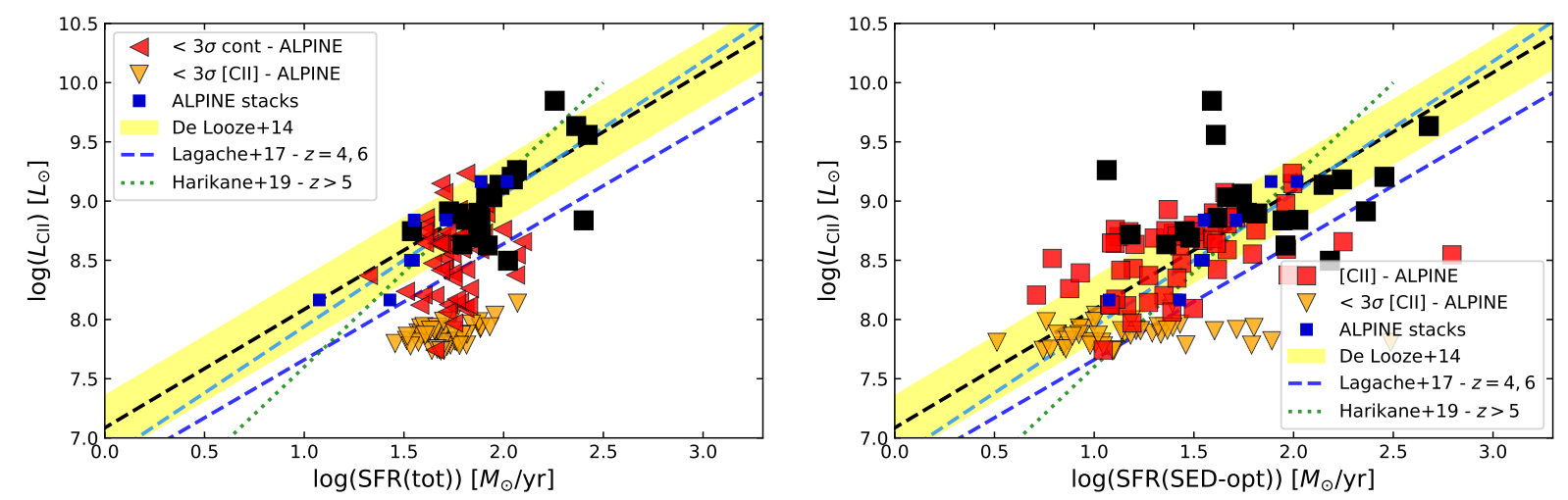

Fig. 3. Same as Fig. 2. Left: SFR(UV+IR) where the IR contribution now includes the 1- $\sigma$ limit on $L_{\mathrm{IR}}$. Right: using the SFR derived from SED fitting of the stellar emission (rest-UV to optical).

are mostly in the range of SFR(tot) $\lesssim 40-100 M_{\odot} \mathrm{yr}^{-1}$. Clearly, tighter constraints are desirable to examine if/how the high- $z$ galaxies do or do not deviate from the local [C II]-SFR relation. Fitting the SED provides one way to account for this. Using tihe results from the multi-band SED fitting results discussed in Faisst et al. (2020) and assuming a Calzetti attenuation law, reduces the apparent $[\mathrm{C} \mathrm{II}]$ excess found when not accounting for dust-obscured star formation. As shown in Fig. 3 (right), the mean offset between the local $L([\mathrm{CII}])-$ SFR relation and the ALPINE data ([C II] detections only) is then reduced to 0.06 dex, although the scatter around the local relation is quite large ( 0.40 dex $)$. However, we note that a comparison using SEDbased SFR values with the relations established by De Looze et al. (2014) would be methodologically inconsistent, since these authors use simple (UV and IR) SFR calibrations, whereas SED fitting allows for varying star formation histories, different ages, etc., which may not yield compatible results and is known to give a larger scatter (see e.g. Wuyts et al. 2011; Schaerer et al. 2013). In any case, all the methods illustrated here show that most, if not all of the $z \sim 4-6$ galaxies included in the ALPINE sample must suffer from some dust attenuation.

\subsection{The [C $I]-S F R$ relation accounting for hidden SF in $z \sim 4-6$ galaxies}

We now proceed to account for hidden SF in all individual ALPINE galaxies in the best possible and consistent way to compare the $[\mathrm{C}$ II $]-S F R$ relation with lower redshift data. To do this, we used the average IRX $-\beta$ relation derived by Fudamoto et al. (2020) for the ALPINE sample from median stacking of the continuum images in bins of the UV slope $\beta$. These authors found an IRX $-\beta$ relation, which is close to but below the relation expected for the SMC attenuation law, with little evolution across the redshift range of the ALPINE sample. We applied their mean IRX- $\beta$ relations to each individual source for which the dust continuum had not been detected, thus yielding a predicted $L_{\mathrm{IR}}{ }^{5}$ and hence a corresponding SFR(IR), using the same assumptions as for the rest of the sample. For continuum-detected galaxies, we used the standard SFR(IR) values, shown above.

The result is illustrated in Fig. 4, showing a very small offset from the local relation $(-0.05 \mathrm{dex})$ and a scatter of $0.28 \mathrm{dex}$ around it (for the [C II] detections). In other words, taking into account a relatively small correction for hidden SF, which is compatible with our continuum non-detections and the IRX- $\beta$

\footnotetext{
5 We note that the $L_{\mathrm{IR}}$ values predicted in this way are below the conservative upper limits determined from the individual non-detections.
}

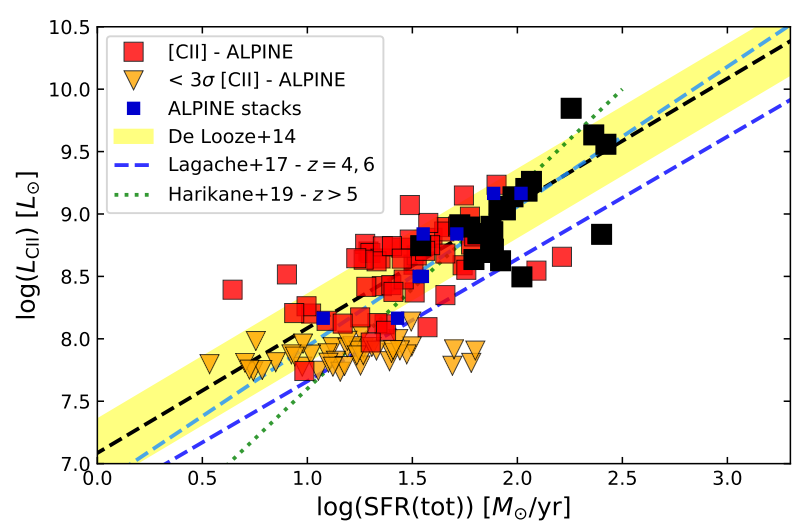

Fig. 4. Same as Fig. 2 using SFR(UV+IR). Here, SFR(IR) is derived from the observed UV slope and luminosity using the ALPINE IRX$\beta$ relation obtained from stacking (Fudamoto et al. 2020). The [C II]detected galaxies follow the local relation well.

relation, the ALPINE [C II]-detected galaxies nicely follow the same $L([\mathrm{CII}])-$ SFR relation as low-redshift galaxies.

Now, if we include the "“agressive" [C II] non-detections and fit the data with a linear relation of the form

$$
\log \left(L([\mathrm{CII}]) / L_{\odot}\right)=a+b \times \log \left(\mathrm{SFR} / M_{\odot} \mathrm{yr}^{-1}\right) \text {, }
$$

using a Bayesian fit including censored data ${ }^{6}$, we obtain a slope that is marginally steeper than the local relation $(1.17 \pm 0.12$, the results from different fits are given in the appendix). However, adopting more conservative upper limits for $L([\mathrm{CII}])$, for example the "secure" limits 7 from Bethermin et al. (2020), which are typically less deep by a factor of two, our fits including censored data yields a slope of $0.96 \pm 0.09$, compatible with unity, and slightly offset (by $\sim-0.03$ dex) with respect to the local relation. From this we conclude that main sequence galaxies at $z \sim 4-6$ may show the same $L([\mathrm{CII}])-$ SFR relation as low redshift galaxies or a relation which is somewhat steeper (with an exponent 1.2). More firm statements are difficult to make at the present stage, until [C II] non-detections and the exact amount of dustobscured star formation are better quantified.

\footnotetext{
6 We follow the method of Kelly (2007) implemented in the python package linmix, https://github.com/jmeyers314/linmix. The method allows for uncertainties in one quantity, here $L([\mathrm{CII}])$.

7 Secure limits are calculated by summing the $3 \sigma$ rms of the noise to the highest flux measured in 1 arcsec around the phase centre in visibility-tapered velocity-integrated flux maps (see Bethermin et al. 2020 for more details).
} 


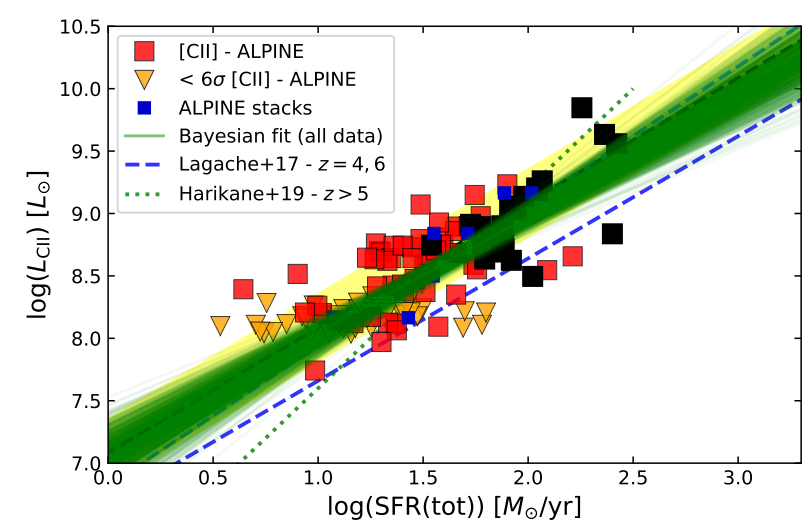

Fig. 5. Same as Fig. 4 for ALPINE sources, but adopting conservative upper limits for the [C II] non-detections (two times the agressive $3 \sigma$ upper limits). The Bayesian linear fit to all the measurements (detections and upper limits) is shown by the dark green lines/band, which also illustrates the probability distribution of the fit. The fit yields a slope of $0.96 \pm 0.09$, compatible with unity, and a small but insignificant offset (by $\sim-0.03 \mathrm{dex}$ ) with respect to the local relation.

\subsection{Is there a universal behaviour of $L([\mathrm{CII})$ at $z>4$ ?}

We now examine how the ALPINE [C II] measurements of $z \sim$ 4-6 galaxies compare with the other available observations at even higher redshifts. To do this, we use the recent compilation of Matthee et al. (2019), which includes 25 reported ALMA [C II] observations of galaxies with known spectroscopic redshifts between $z=6.0$ and $z=7.212$. Importantly, Matthee et al. (2019) recomputed [C II] non-detection limits using empiricallymotivated $\left[\mathrm{C}\right.$ II] line widths ${ }^{8}$. Furthermore they have uniformly re-derived SFR(UV) and SFR(IR) from the observations, assuming a modified black body with $T_{\mathrm{d}}=45 \mathrm{~K}$. We use their derived properties, after rescaling them to the IMF and SFR(IR) calibrations adopted in this paper (see Sect. 2). To this we add 11 measurements ( 6 detections, 5 upper limits) of galaxies between $z=6.0$ and 9.11 from Harikane et al. (2020), who report three new observations and eight others not included in the Matthee et al. (2019) compilation. For consistency, we used the SFR(UV) and SFR(IR) values, and we carefully rescaled their results to a single, consistent IMF and to the same SFR(IR) calibration. Finally, for sources that are not detected in the dust continuum, we corrected for hidden SF by applying the IRX- $\beta$ relation derived at $z \sim 5.5$ from the ALPINE sample when the UV slope is reported. For the majority of the galaxies, the correction turns out to be small, since their UV slope is fairly blue. The data are plotted Fig. 6.

Interestingly, most of the known $z \gtrsim 6$ galaxies largely follow the same behaviour/trend as the ALPINE galaxies. At SFR(tot) $\gtrsim 30 M_{\odot} \mathrm{yr}^{-1}$, very few points deviate by more than $1 \sigma$ from the De Looze et al. (2014) relation. Differences between our results and those shown in Harikane et al. (2020) are explained by several effects: by our use of a single IMF consistent with the De Looze et al. (2014) relation, a consistent use of calibrated SFR determinations for all sources (no SEDbased SFR as for some of their sources) following Matthee et al. (2019), and finally by the adoption of conservative line widths to determine upper limits on $L([\mathrm{CII}])$.

[C II] is undetected in a significant number of galaxies at $\mathrm{SFR} \lesssim 30 M_{\odot} \mathrm{yr}^{-1}$. Assuming a $F H W M=150 \mathrm{~km} \mathrm{~s}^{-1}$ for the

\footnotetext{
8 They use $F W H M=-1215-66 \times M_{\mathrm{UV}} \mathrm{km} \mathrm{s}^{-1}$, translating to a minimum $F W H M=123 \mathrm{~km} \mathrm{~s}^{-1}$ for the UV-faintest [C II] non-detected galaxy in their sample (Matthee, priv. comm.).
}

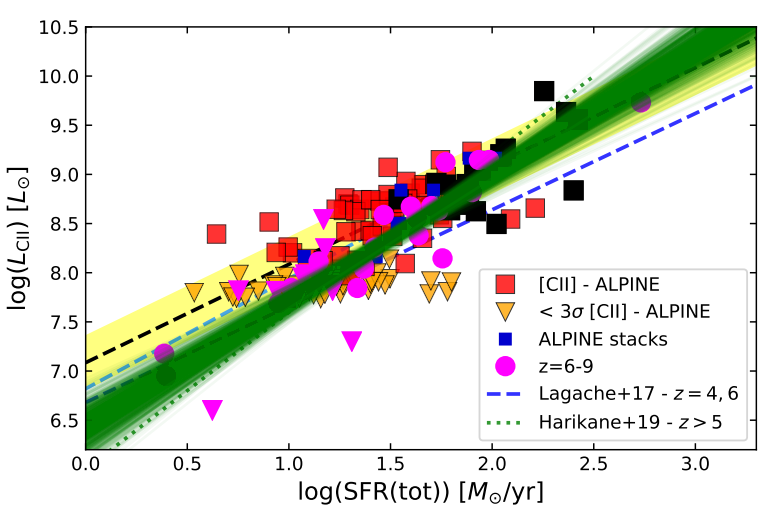

Fig. 6. $[\mathrm{C}$ II $]-S F R$ relation combining the ALPINE sample and observations of $z \sim 6-9$ galaxies taken from the literature. The $z>6$ data, after proper uniformisation, are plotted with pink symbols (circles for detections, and triangles for upper limits). The SFR of all sources includes an SFR(IR) contribution, determined from the observed dust continuum or from the IRX $-\beta$ relation if undetected. All [C II] non-detections are illustrated by triangles, showing $3 \sigma$ (aggressive) upper limits. The Bayesian linear fit to all the $z>4$ measurements (detections and upper limits) is shown by the dark green lines/band. The slope is somewhat steeper than unity: $1.28 \pm 0.10$.

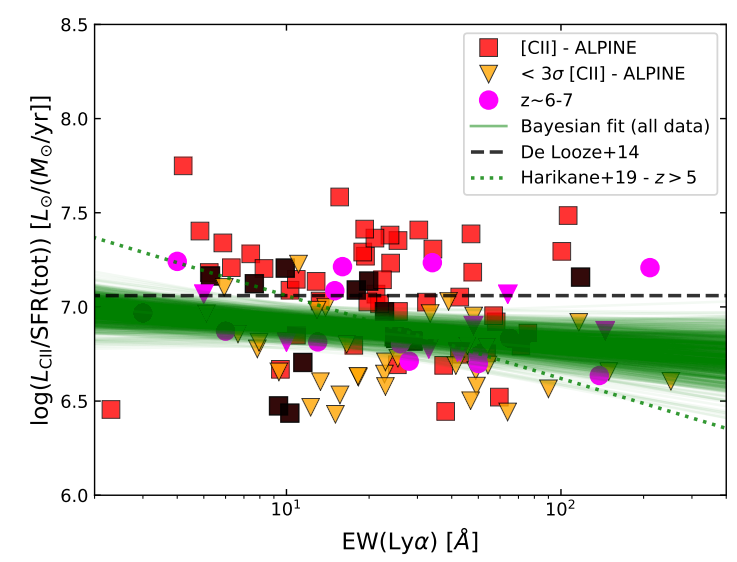

Fig. 7. $L([\mathrm{C} \mathrm{II}]) / \mathrm{SFR}$ (tot) as a function of the rest-frame $\mathrm{Ly} \alpha$ equivalent width of the ALPINE sources and $z \sim 6-7$ galaxies taken from the literature (pink symbols: circles for detections, and triangles for upper limits). The dotted line shows the fitting relation obtained by Harikane et al. (2018) for a compilation of $z \sim 5.7-7$ galaxies, the horizontal line the average value from the local relation. The green lines/band show the fit to the data; the resulting slope is $-0.11 \pm 0.06$, indicating a weak dependence on $\operatorname{EW}(\operatorname{Ly} \alpha)$.

ALPINE sources and the 15 upper limits from the $z>6$ data discussed above, we find that the deepest $3 \sigma$ upper limits are $\log \left(L([\mathrm{CII}]) / L_{\odot}\right)<7.8$ for the bulk of the data. While a fraction of those are well within the scatter around the "local" De Looze et al. (2014) relation, several are probably below this, which pushes the average $L([\mathrm{CII}]) / \mathrm{SFR}$ ratio of both the ALPINE sample and the full high- $z$ galaxy sample to $\log (L([\mathrm{CII}]) /) \mathrm{SFR} \approx 6.85 L_{\odot} / M_{\odot} \mathrm{yr}^{-1}$, approximately 0.2 (0.1) dex lower than the reference value from De Looze et al. (2014) for the $\mathrm{H} \mathrm{II} /$ starburst (complete) galaxy samples. Two non-detections at $\mathrm{SFR}<30 M_{\odot} \mathrm{yr}^{-1}$ are strongly underluminous in [C II] compared to the rest of the sample: these are two lensed galaxies at $z>8$, A2744-YD4 at $z=8.382$ and MACS1149-JD1 at $z=9.11$ observed by Laporte et al. (2019), which were previously detected by ALMA in the [O III] $88 \mu \mathrm{m}$ line by Laporte et al. (2017) and Hashimoto et al. (2018). On the other hand, 
another [O III]-detected lensed $z=8.312$ galaxy (MACS0416Y1 from Tamura et al. 2019) is detected in [C II] and follows the observed trend well.

To quantify this behaviour again, we use the Bayesian fit including censored data. The results including the uncertainties and upper limits on $L([\mathrm{CII}])$ are shown by the green lines/band in Fig. 6. The fit shows that the inclusion of the upper limits primarily leads to a somewhat steeper (super-linear) slope $(1.28 \pm 0.1)$ in the $L([\mathrm{CII}])-$ SFR relation for galaxies at $z>4$, and to an overall (but slight) decrease of the normalisation, for instance to a lower [C II] luminosity on average at a given SFR, as already mentioned and shown in Fig. 7. Overall, the observational data shows a behaviour that is quite comparable to the mean $L([\mathrm{CII}])-$ SFR relations predicted by the models of Lagache et al. (2018) between $z=4$ and $z=6$. The fit to the data shown in Fig. 6 is also similar to the mean relation obtained from the recent simulations of high- $z$ galaxies by Arata et al. (2020), who find a somewhat steeper slope of 1.47. In contrast, the cosmological plus radiative transfer simulations of Leung et al. (2020) for $z \sim 6$ galaxies predict a $L([\mathrm{CII}])-S F R$ relation with, on average, significantly lower [C II] luminosities than the observational data shown here. Furthermore, the slope of their best-fit relation $(0.66 \pm 0.01)$ is flatter than unity and than those found in our study.

Interestingly, with the enlarged sample (ALPINE and $z>6$ galaxies) our fits yield slopes steeper than unity using both options for the [C II] upper limits (agressive versus conservative; cf. above). This result is mostly driven by a few additional data points at low $L([\mathrm{CII}])$ and low SFR, which have low uncertainties on $L([\mathrm{CII}])$ and thus a fairly strong leverage. Whether these points are truly representative of the bulk of the population of fainter galaxies or "“outliers" remains to be confirmed with new observations probing this regime.

Overall, we conclude that the $[\mathrm{CII}]$ measurements (detections and upper limits) of star-forming galaxies at $z \sim 4-8$ follow a unique relation between $L([\mathrm{CII}])$ and $\mathrm{SFR}(\mathrm{UV})+\mathrm{SFR}(\mathrm{IR})$ quite well over nearly two orders of magnitude in the [C II] luminosity. This holds for a wide variety of galaxy types (LAEs, LBGs primarily, plus two SMGs from Marrone et al. 2018 included in the Harikane et al. 2020 compilation) issued from different selections. Taking the [C II] non-detections into account, the $L([\mathrm{CII}])-$ SFR rrelation appears to be somewhat steeper and offset from the "local" counterpart determined by De Looze et al. (2014). Whether the relation shows a turnover below SFR $\lesssim 10-30 M_{\odot} \mathrm{yr}^{-1}$, as suggested, for example by Matthee et al. (2019), cannot be established from the available data. This would require more sensitive measurements.

\subsection{Is there a dependence of [C $॥]$ with the Lyo equivalent width in high-z galaxies?}

Several authors have pointed out that galaxies with an increasing $\mathrm{Ly} \alpha$ equivalent width show a fainter [C II] emission, compared to expectations from the local [C II]-SFR relation (see e.g. Carniani et al. 2018; Harikane et al. 2018, 2020). They relate this trend to a possible increase of the ionisation parameter or to a low covering fraction of photodissociation regions with increasing $\operatorname{Ly} \alpha$ emission. Benefitting now from the large amount of new data from ALPINE for which we also have $\operatorname{EW}(\operatorname{Ly} \alpha)$ measurements (for 58 [C II]-detected plus 33 non-detected sources, taken from Cassata et al. 2020), we show the behaviour of $L([\mathrm{CII}]) / \mathrm{SFR}$ (tot) as a function of $\operatorname{EW}(\operatorname{Ly} \alpha)$ in Fig. 7. The ALPINE sources cover a wide range of Ly $\alpha$ equivalent widths, also including relatively large EWs, some of which were selected as LAE. Again, a large scatter is found in $L([\mathrm{CII}]) / \mathrm{SFR}$ at all values of $\operatorname{EW}(\operatorname{Ly} \alpha)$, and the fit to all the data including the non-detections shows a weak dependence of $\operatorname{EW}(\operatorname{Ly} \alpha)$ on $L([\mathrm{CII}]) / \mathrm{SFR}^{9}$. Clearly, we cannot claim a strong anti-correlation of [C II] with increasing Ly $\alpha$ equivalence width, in contrast to Harikane et al. (2020). The difference compared to their work lies in our significantly larger dataset, our use of "uniformised" values for SFR, and the use of a more conservative line width for the determination of the upper limits on [C II] luminosities, as already mentioned above.

\section{Observed [ $\left.\mathrm{C}_{\mathrm{II}}\right] 158 \mu \mathrm{m}$ line to IR continuum ratios}

With the exception of some lensed sources from the SPT survey (Gullberg et al. 2015), the $z>3$ galaxies currently detected in the dust continuum have typical (lensing-corrected, if applicable) IR luminosities in the range of $L_{\mathrm{IR}} \gtrsim 10^{11}$ to $2 \times$ $10^{12} L_{\odot}$, hence they are LIRG or ULIRG by definition. This is also the case for the continuum-detected ALPINE sources (see Bethermin et al. 2020). In this regime of high-IR luminosities, low- $z$ galaxies show the well-known "[C II] deficit", meaning a drop of $L([\mathrm{CII}]) / L_{\mathrm{IR}}$ towards high $L_{\mathrm{IR}}$ (see e.g. Malhotra et al. 2001; Graciá-Carpio et al. 2011). It is therefore of interest to examine how high redshift galaxies, the ALPINE sample in particular, and others, behave in this respect.

Since the IR continuum is undetected in many observations of normal star-forming galaxies at high redshift, we first plot the $L([\mathrm{CII}]) / L_{\mathrm{IR}}$ ratio as a function of the $[\mathrm{C} \mathrm{II}]$ luminosity instead of $L_{\mathrm{IR}}$. The result is shown in the left panel of Fig. 8, where we show the data for all the [C II]-detected galaxies of ALPINE, the $z>6$ data from the compilation of Matthee et al. (2019), other [C II] detections (non-AGN-dominated sources) at $z>3$ taken from the compilation in Gullberg et al. (2015), the SPT sources of Gullberg et al. (2015), and observations at $z<3$ from the compilation of Zanella et al. (2018). We note that we use the total IR luminosity here (from 8 to $1000 \mu \mathrm{m}$ ), following Zanella et al. (2018), for example, whereas other authors use the farIR luminosity, $L_{\mathrm{FIR}}$ (from 40 to $122 \mu \mathrm{m}$ restframe), as a reference; in the Zanella et al. (2018) compilation, one typically has $L_{\mathrm{IR}} / L_{\mathrm{FIR}}=1.6$. For the SED template used for ALPINE one has $L_{\mathrm{IR}} / L_{\mathrm{FIR}}=1.628$.

The ALPINE sources detected in the continuum show a ratio $L([\mathrm{CII}]) / L_{\mathrm{IR}} \sim(1-3) \times 10^{-3}$ (or a factor of 1.628 higher when compared to $L_{\mathrm{FIR}}$ ), comparable to the "normal" $z<$ 1 sources, whereas the IR luminous [C II]-deficient galaxies have $L([\mathrm{CII}]) / L_{\mathrm{IR}}<10^{-3}$. The same is also found for the other continuum-detected $z>6$ galaxies, and the majority of the $z \sim 4-7$ sources that are currently undetected in the dust continuum are also compatible with normal or higher $L([\mathrm{CII}]) / L_{\mathrm{IR}}$ ratios. In other words, the majority of the $z>4$ galaxies where [C II] is detected do not seem to show a deficit in $L([\mathrm{CII}]) / L_{\mathrm{IR}}$, similar to earlier findings at lower redshift (e.g. $z \sim 1-2$, Zanella et al. 2018). On the other hand, the SPT sample, which is significantly brighter than the ALPINE sources and the $z>6$ LBGs and LAEs, shows several sources with $L([\mathrm{CII}]) / L_{\mathrm{IR}}<10^{-3}$ and an increasing [C II]-deficit at IR luminosities above $\gtrsim 10^{12} L_{\odot}$, as shown by Gullberg et al. (2015). One may therefore speculate that a [C II] deficit is also present in high- $z$ galaxies, albeit at intrinsically higher IR luminosities, again suggesting that the [C II]/IR-deficit is not a universal property, as already suggested earlier (cf. Zanella et al. 2018).

9 The Bayesian fit yields $\log (L([\mathrm{CII}]) / \mathrm{SFR})=(6.99 \pm 0.07)-(0.10 \pm$ $0.06) \times \log (\mathrm{EW})$ in the units plotted in Fig. 7 . 

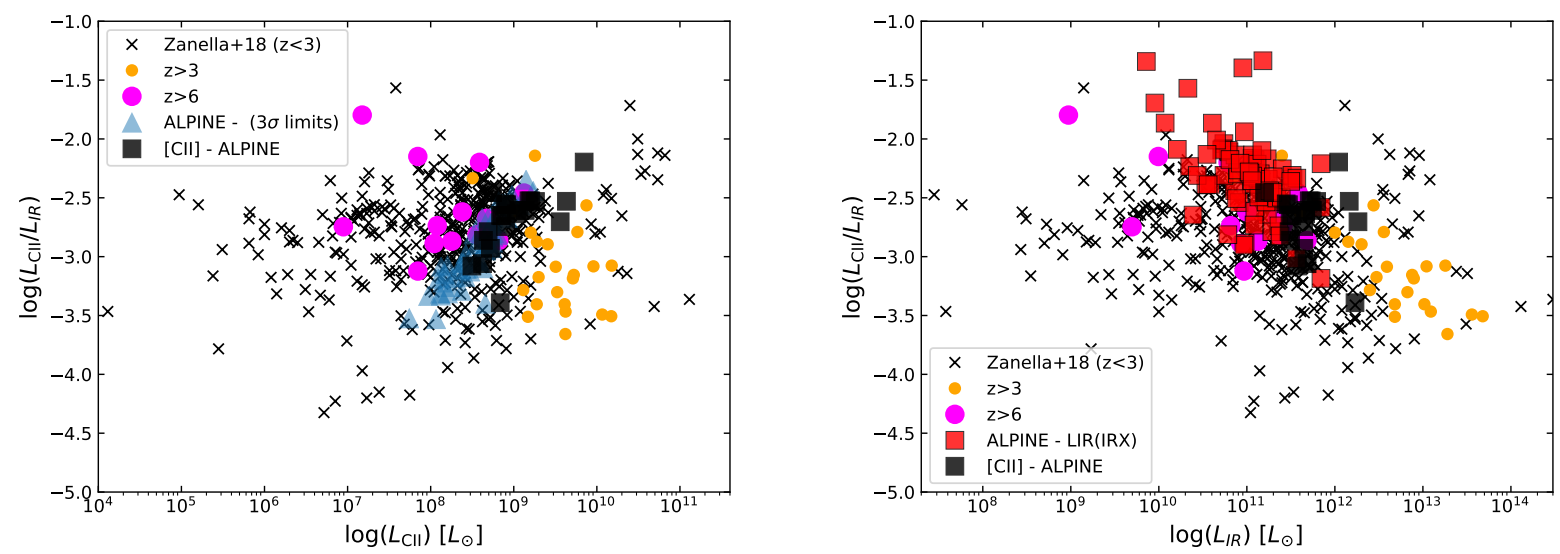

Fig. 8. $L([\mathrm{CII}]) / L_{\mathrm{IR}}$ versus $L([\mathrm{CII}])$ (left panel) and $L_{\mathrm{IR}}($ right) for the ALPINE sources and comparison samples where the [C II] line is detected. For the $z>6$ sources, taken from the Matthee et al. (2019) sample, we adopt $T_{\mathrm{d}}=45 \mathrm{~K}$ for the detections and $L_{\mathrm{IR}}$ upper limits. For galaxies not detected in the dust-continuum, we show their $L([\mathrm{CII}]) / L_{\mathrm{IR}} 3 \sigma$ lower limit in the left panel as triangles. Right panel: $L_{\mathrm{IR}}$ limits of the ALPINE sources have been replaced by the $L_{\mathrm{IR}}$ values computed from the IRX $-\beta$ relation, where possible.

In the right panel of Fig. 8, we show a more classical version of the dependence of the $L([\mathrm{CII}]) / L_{\mathrm{IR}}$ ratio, plotted as a function of $L_{\mathrm{IR}}$, where the IR luminosity of the high- $z(z \sim 4-6$ galaxies from ALPINE and the $z>6$ sample) is taken from the observations or has been computed from the ALPINE IRX $-\beta$ relation (as used in Figs. 4-7 and 5) for the continuum non-detected sources. Most of the latter sources have predicted $L_{\mathrm{IR}} \sim 10^{10}-$ $\left(3 \times 10^{11}\right) L_{\odot}$, and $L([\mathrm{CII}]) / L_{\mathrm{IR}}$ ratio ranges between $10^{-3}$ and $10^{-2}$, compatible with the bulk of the $z<3$ galaxies.

Since the observed decrease of $L([\mathrm{CII}]) / L_{\mathrm{IR}}$ in low and high- $z$ galaxies is known to correlate with the increasing dust temperature (e.g. Malhotra et al. 2001; Graciá-Carpio et al. 2011; Diaz-Santos et al. 2013; Gullberg et al. 2015), one might be tempted to conclude that the "normal" $L([\mathrm{CII}]) / L_{\mathrm{IR}}$ ratio found for the majority of the ALPINE galaxies and $z>6 \mathrm{LBGs}$ and LAEs, could indicate that these sources do not harbor particularly hot dust. In the context of the intensely debated uncertainties on the typical dust temperatures of normal galaxies in the early Universe (see e.g. Bouwens et al. 2016; Faisst et al. 2017; Ferrara et al. 2017), this would have important implications. Conversely, from the analysis of Magdis et al. (2014) one would expect some [C II] deficiency for the ALPINE continuumdetected galaxies at $T_{\mathrm{d}} \sim 42 \mathrm{~K}$ of our template, but this effect is not seen in our data. In any case, Fig. 8 should not be overinterpreted since the inferred IR luminosity itself depends on the assumed IR SED template, meaning directly or indirectly on $T_{\mathrm{d}}$. Independent constraints on the IR SED and dust temperature of high- $z$ galaxies are clearly needed.

\section{Discussion}

If star formation were unobscured in most of the $z \sim$ 4-6 galaxies covered by the ALPINE survey, our observations would indicate that [C II] is over-luminous at a given $\mathrm{SFR} \approx \mathrm{SFR}(\mathrm{UV})$, compared to the observed correlation for low redshift galaxies (see Fig. 2). At face value, such a conclusion would be quite in contrast with earlier studies of $z>6$ galaxies, which for example have argued that [C II] was less luminous than expected from comparisons with the low- $z$ reference sample (see e.g. Ouchi et al. 2013; Bradač et al. 2017; Harikane et al. 2018).

However, as argued above, it seems much more likely that a fraction of the UV light from star formation is attenuated by dust, in the majority of our targets, as well as in those from which we do not detect dust-continuum emission with ALMA. Indeed, a relatively small correction of SFR(UV) - upward by a factor of $\sim 2$ on average - is sufficient to bring the [C II] measurements on average into agreement with the local $L([\mathrm{CII}])-$ SFR relation (cf. Sect. 3.2). The amount of this correction appears very reasonable from several points of view: firstly, it corresponds to the average correction obtained from multi-band SED fitting of the rest-UVto-optical SED; and secondly, the same correction is found, on average, by applying an empirically calibrated IRX- $\beta$ relation of the ALPINE galaxies derived from stacking to the individual ALPINE galaxies, which are not detected in the dust continuum. Finally, stacking the continuum in bins of $L([\mathrm{CII}])$ also indicates a necessary correction to the SFR(UV) as shown by Bethermin et al. (2020), leading to a fair agreement of the stacked data with the local relation.

Taking into account a correction for dust-obscured star formation, we then examined and derived the empirical relation between $L([\mathrm{CII}])$ and the total SFR(tot) for $z>4$ galaxies, using both the ALPINE sample covering $z \sim 4-6$ and data from the literature for $z \sim 6-9$ galaxies. We also included [C II] non-detections in a Bayesian linear fit of the data (Sect. 3.3 and appendix). We also stress the importance of a consistent use of SFR calibrations, IMF normalisations, and empirically motivated [C II] line widths to compute upper limits (see also Matthee et al. 2019), which must be taken into account for meaningful and consistent comparisons of different datasets and to establish, for example, a possible evolution of the $L$ ([CII])-SFR relation with redshift. Some of our results are obviously also subject to uncertainties and future improvements, which we now briefly discuss.

Making reasonable assumptions on the dust-obscured SFR and using for the first time a large sample of up to 150 galaxies, we have shown that the [C II] luminosity of high- $z(z>4)$ galaxies correlates well with the total SFR, over approximately two orders of magnitude in SFR. The data is described well by a linear relationship between $\log (L([\mathrm{CII}]))$ and $\log \left(\mathrm{SFR}_{\text {tot }}\right)$ with a slope close to unity $(b \sim 0.8-1.3)$ (see Table A.1). However, the exact slope of the relation depends in part on the [C II]undetected sources, and hence on the detailed assumptions on the upper limits, which depends not only on assumed line widths, but also on the hypothesis about size (point-like or slightly extended sources). Deeper observations for some of the ALPINE targets would be easily attainable with ALMA, and helpful to better understand the sources with $\log (L([\mathrm{CII}])) \lesssim 10^{8} L_{\odot}$. To firm up the result of a possibly steeper $L([\mathrm{CII}])-$ SFR relation at high- $z$ than for local galaxies, it is also clearly important to acquire more measurements of fainter galaxies with lower star 
formation rates, ideally at SFR $\lesssim 1-3 M_{\odot} \mathrm{yr}^{-1}$, where currently only very few observations of lensed galaxies have been obtained (Knudsen et al. 2016; Bradač et al. 2017).

Although we fitted the available data with simple linear relations (i.e. a power-law dependence of $L([\mathrm{CII}])$ on SFR), nature may be more complicated, and the conditions may be different in high-redshift galaxies. The high- $z$ data discussed here do not allow us to exclude different behaviour at low SFR or low $L([\mathrm{CII}])$, as suggested, for example, by Matthee et al. (2019). However, on resolved scales in our Galaxy and for individual galaxies from the nearby Universe up to $z \sim 1-3$, different studies have empirically established a correlation between [C II] and the total SFR with simple power laws with exponents of 0.8-1.2 extending over approximately six orders of magnitude (see e.g. Pineda et al. 2014; De Looze et al. 2011, 2014; Zanella et al. 2018), and which include the range probed by high- $z$ observations. From an empirical point of view and in the absence of strongly deviating data, we did not consider other functional forms of the [C II]-SFR relation.

Beyond [CII], the second fundamental quantity for this work is obviously the total SFR, which is currently not easy to determine, due to technical limitations (insufficient sensitivity to detect dust-continuum emission) and our limited knowledge of the dust properties and IR template, which are required to infer the total IR luminosity, and hence the dust-obscured part SFR(IR). On the other hand, SFR(UV) is easy to determine for the galaxies of interest here, since all of them were previously detected at these wavelengths for our survey (Le Fèvre et al. 2020). The IR template used in our work to translate the restframe $158 \mu \mathrm{m}$ continuum measurements into the total $L_{\mathrm{IR}}$ has a similar "bolometric correction" to a modified black body (MBB) with $T_{\mathrm{d}} \approx 42 \mathrm{~K}$ (Bethermin et al. 2020). Using, for example, the empirical template of Schreiber et al. (2018) would imply $L_{\mathrm{IR}}$ values that are higher by $43 \%$, comparable to an MBB with $T_{\mathrm{d}} \sim 45 \mathrm{~K}$. If even higher dust temperatures were appropriate, $L_{\mathrm{IR}}$ would, for example, increase by a factor of 1.87 (3.79) for $T_{\mathrm{d}}=50$ (60) K compared to Bethermin et al. (2020).

With our assumptions and the adopted IRX- $\beta$ correction, for the ALPINE galaxies one has $\mathrm{SFR}(\mathrm{UV}) \approx \mathrm{SFR}(\mathrm{IR})$ on average, and most galaxies have $\mathrm{SFR}(\mathrm{UV}) \lesssim 2 \mathrm{SFR}(\mathrm{IR})$. In this case, an increase of $L_{\mathrm{IR}}$ by a factor of two (3) would translate as an increase of the total SFR by a factor of 1.5-1.6 (2-2.3). This effect could thus shift the [C II]-SFR relation by this amount, away from the local relation. Whether (and by how much) this could also change the slope of the relation depends if the dustobscured SFR fraction is constant in all galaxies, and how the dust temperature may vary with galaxy properties, all of which are largely unknown for high- $z$ galaxies.

Clearly, accurately determining the total SFR of high- $z$ galaxies will lead to significantly more robust results on the [C II]-SFR relation in the distant Universe. Efforts are under way to constrain the dust temperatures at high-z (e.g. Hirashita et al. 2017; Faisst et al. 2017; Bakx et al. 2020). Alternatively, the JWST should soon provide measurements of rest-optical lines including hydrogen recombination lines, which will allow one to determine, for example, the $\mathrm{H} \alpha$ SFR and dust corrections using the Balmer decrement for high- $z$ galaxies. This could become an important and complementary method to nail down some of the uncertainties discussed here, and indirectly also to constrain the dust temperature and $L_{\mathrm{IR}}$ of distant galaxies.

Finally, we would like to caution that the [C II] luminosity may not necessarily trace the SFR accurately in general, especially in high redshift galaxies. Although $L([\mathrm{CII}])$ empirically correlates well with the SFR, the main physical reason(s) for this dependence are not well understood and predictive models are therefore difficult to construct, presumably largely since [C II] is known to originate from a broad range of ISM phases and regions with different conditions (see e.g. Vallini et al. 2015; Lagache et al. 2018; Ferrara et al. 2019; Popping et al. 2019). In fact, the empirical correlations of $L([\mathrm{CII}])$ determined here and in earlier studies are with the UV+IR luminosity, or a combination of the two, which can be converted to the SFR if one assumes a particular star formation history and age of the population. More fundamentally, the data thus probably indicate a correlation of the [C II] luminosity with the intrinsic UV luminosity of the galaxy - part of which emerges in the UV, and the other part after processing by dust in the IR - which is also physically significant, since [C II] requires photons capable of singly ionising carbon atoms, that is to say with energies $>11.26 \mathrm{eV}$ (wavelength $<1102 \AA$ ). This implies in particular that $L([\mathrm{CII}])$ does not need to closely follow the instantaneous SFR in galaxies with strongly varying (irregular, burst, etc.) star formation histories, where significant variations between $L_{\mathrm{UV}}$ and the SFR are expected (see e.g. Schaerer et al. 2013; Madau \& Dickinson 2014). Such situations are probably more common in the early Universe, and one may therefore expect a better correlation of $L([\mathrm{CII}])$ with the intrinsic (total) UV luminosity than with other tracers of the SFR, such as $\mathrm{H}$ recombination lines.

Furthermore, [C II] emission may also depend on metallicity and other galaxy properties such as the gas fraction, distance from the main sequence, and so on (cf. Vallini et al. 2015; Lagache et al. 2018; Zanella et al. 2018). Currently, these quantities are largely unknown for high- $z$ galaxies and are difficult to measure. In addition, the ALPINE sample is, by construction, not well placed to examine possible dependences on the main sequence distance, since it selected main sequence galaxies and subtle variations from it are difficult to measure. Future independent measurements may yield a more refined picture of the main processes governing the emission of [C II] in distant galaxies.

Other results from the rich ALPINE dataset are presented elsewhere. Beyond those already mentioned earlier, these cover, for example, the use of [C II] to estimate the amount of gas in galaxies, morphological studies, the detection of [C II] in the circumgalactic medium, studies of the kinematics between [C II] and $\operatorname{Ly} \alpha$, the discovery of very obscured sources, and several other topics. For more details on these issues, see DessaugesZavadsky et al. (2020), Fujimoto et al. (2020), Ginolfi et al. (2020), Cassata et al. (2020), Romano et al. (2020).

\section{Conclusions}

We analysed the new [C II] $158 \mu \mathrm{m}$ measurements from the ALPINE survey of star-forming galaxies at $z \sim 4-6$ (Le Fèvre et al. 2020; Bethermin et al. 2020; Faisst et al. 2020), which for the first time provides a large sample (118 galaxies) to study [C II] emission and its correlation with the star formation rate at high redshift. We examined whether our data and other observations at $z>6$ - now totalling 153 galaxies - are compatible with the observed correlation between the [C II] luminosity and SFR found at lower redshift, and described in the De Looze et al. (2014) reference sample.

To compare the high- $z$ observations to the earlier data, we used consistent SFR calibrations (based on UV and IR continuum luminosities) and a carefully homogenised IMF. We also took into account the $[\mathrm{C} \mathrm{II}]$ non-detections, which are translated into upper limits on $L([\mathrm{CII}])$ adopting empirically motivated assumptions on the [C II] line widths, which we re-examined using our own data and literature data (see Fig. 1). 
The ALPINE galaxies, which are both detected in [C II] and the dust continuum, show a good agreement with the low- $z$ $L([C I I])-S F R$ relation when considering the total SFR(UV+IR). A fraction of the non-detected ALPINE galaxies in the dust continuum appear over-luminous in $L([\mathrm{CII}])$ compared to expectations from the De Looze et al. (2014) relation, when no correction for dust attenuation is made (see Fig. 2, left). This is in contrast with earlier studies, which have often reported apparent deficits of [C II] in high- $z$ galaxies (e.g. Ouchi et al. 2013; Inoue et al. 2016; Harikane et al. 2018). Using the results from two different stacking methods, described in Bethermin et al. (2020), Fudamoto et al. (2020), and SED fits allows us to account for dust-obscured star formation in these galaxies, thus increasing their total SFR by a factor of $\sim 2$ on average, which brings the ALPINE galaxies into agreement with the local [C II]-SFR relation (Figs. 3, right, and 4).

When conservative upper limits from the [C II] non-detected galaxies $(\sim 1 / 3$ of the ALPINE survey) are also considered, we find that $L([\mathrm{CII}])$ scales linearly with the total SFR for the ALPINE sample, although with a slightly lower normalisation $(L([\mathrm{CII}]))$ than the local $\mathrm{HII} /$ starburst galaxy sample of De Looze et al. (2014). Using more agressive upper limits leads to a steepening of the $L([\mathrm{CII}])-$ SFR relation. A steeper increase of $L([\mathrm{CII}])$ with SFR is also found when all the available [C II] measurements (detections and upper limits) at $z \sim 4-8$, including other ALMA measurements from the literature are combined (Fig. 6). Given the remaining uncertainties on the [C II] nondetected galaxies and the exact amount of dust-obscured SFR, we conclude that the exact slope of the $L([\mathrm{CII}])-$ SFR relation at $z>4$ is not firmly established.

Upon analysing the homogenised sample of $153 z>4$ galaxies with [C II] measurements (detections or upper limits), we find that very few galaxies deviate significantly from the bulk of the sample, and that most $z \sim 4-8$ galaxies show an $L([\mathrm{CII}])-$ SFR relation that is not very different from that of low- $z$ galaxies nearly 13 Gyr later. In other words, the currently available data show no strong evidence for a deficit of [C II] from $z \sim 4$ to 8 , in contrast to several earlier results, but in line with other suggestions (Carniani et al. 2018; Matthee et al. 2019). The only strong outliers from the $L([\mathrm{CII}])-$ SFR relation are two galaxies at $z>8$ with [O III] $88 \mu \mathrm{m}$ line detections with ALMA and no [C II] $158 \mu \mathrm{m}$ (Laporte et al. 2019), which may indicate a more fundamental change of properties in the very early Universe.

We also examined the behaviour of $L([\mathrm{CII}]) / \mathrm{SFR}$ with the observed Ly $\alpha$ equivalent width of the ALPINE galaxies and literature data, and we do not find a strong dependence of the [C II] excess or deficiency with $\operatorname{EW}(\operatorname{Ly} \alpha)$ at $z>4$ (Fig. 7), in contrast with earlier suggestions (e.g. Harikane et al. 2018, 2020; Matthee et al. 2019). Finally, we show that the derived ratio $L([\mathrm{CII}]) / L_{\mathrm{IR}} \sim(1-3) \times 10^{-3}$ for the ALPINE sources, comparable to that of "normal" galaxies at lower redshift (Fig. 4).

Overall, our results, using 153 galaxies at $z>4$, suggest that the [C II] luminosity can be used to trace the SFR at these high redshifts, although the scatter is higher than at low redshift, as already indicated by Carniani et al. (2018), for example. Furthermore, there is some evidence for a possible steepening of the $L([\mathrm{CII}])-$ SFR relation compared to $z<3$, although this needs to be confirmed with future measurements and better constraints on dust-obscured star formation in high- $z$ galaxies, which can be obtained with new ALMA and future JWST observations.

Acknowledgements. This paper is based on data obtained with the ALMA Observatory, under Large Program 2017.1.00428.L. ALMA is a partnership of ESO (representing its member states), NSF(USA) and NINS (Japan), together with NRC (Canada), MOST and ASIAA (Taiwan), and KASI (Republic of Korea), in cooperation with the Republic of Chile. The Joint ALMA Observatory is operated by ESO, AUI/NRAO and NAOJ. DS, MG and MD acknowledge support from the Swiss National Science Foundation. AC, CG, FL, FP and MT acknowledge the support from grant PRIN MIUR 2017 - 20173ML3WW_001. EI acknowledges partial support from FONDECYT through grant $\mathrm{N}^{\circ} 117 \overline{1710}$. GCJ and RM acknowledge ERC Advanced Grant 695671 "QUENCH" and support by the Science and Technology Facilities Council (STFC). GL acknowledges support from the European Research Council (ERC) under the European Union's Horizon 2020 research and innovation programme (project CONCERTO, grant agreement No. 788212) and from the Excellence Initiative of Aix-Marseille University-A*Midex, a French "Investissements d'Avenir" programme. DR acknowledges support from the National Science Foundation under grant numbers AST-1614213 and AST-1910107 and from the Alexander von Humboldt Foundation through a Humboldt Research Fellowship for Experienced Researchers. ST acknowledges support from the ERC Consolidator Grant funding scheme (project ConTExT, grant No. 648179). The Cosmic DAWN Center is funded by the Danish National Research Foundation under grant No. 140 LV acknowledges funding from the European Union's Horizon 2020 research and innovation program under the Marie Sklodowska-Curie Grant agreement No. 746119. This paper is dedicated to the memory of Olivier Le Fèvre, PI of the ALPINE survey.

\section{References}

Arata, S., Yajima, H., Nagamine, K., Abe, M., \& Khochfar, S. 2020, MNRAS, 498, 5541

Bakx, T. J. L. C., Tamura, Y., Hashimoto, T., et al. 2020, MNRAS, 493, 4294

Bethermin, M., Fudamoto, Y., Ginolfi, M., et al. 2020, A\&A, 643, A2

Bouwens, R. J., Aravena, M., Decarli, R., et al. 2016, ApJ, 833, 72

Bradač, M., Garcia-Appadoo, D., Huang, K.-H., et al. 2017, ApJ, 836, L2

Capak, P. L., Carilli, C., Jones, G., et al. 2015, Nature, 522, 455

Carilli, C., Murphy, E., Ferrara, A., \& Dayal, P. 2017, ApJ, 848, 49

Carniani, S., Maiolino, R., Amorin, R., et al. 2018, MNRAS, 478, 1170

Cassata, P., Morselli, L., Faisst, A., et al. 2020, A\&A, 643, A6

Chabrier, G. 2003, PASP, 115, 763

De Looze, I., Baes, M., Bendo, G. J., Cortese, L., \& Fritz, J. 2011, MNRAS, 416, 2712

De Looze, I., Cormier, D., Lebouteiller, V., et al. 2014, A\&A, 568, A62

Dessauges-Zavadsky, M., Ginolfi, M., Pozzi, F., et al. 2020, A\&A, 643, A5

Diaz-Santos, T., Armus, L., Charmandaris, V., et al. 2013, ApJ, 774, 68

Fahrion, K., Cormier, D., Bigiel, F., et al. 2017, A\&A, 599, A9

Faisst, A. L., Capak, P. L., Yan, L., et al. 2017, ApJ, 847, 21

Faisst, A. L., Schaerer, D., Lemaux, B. C., et al. 2020, ApJS, 247, 61

Ferrara, A., Hirashita, H., Ouchi, M., \& Fujimoto, S. 2017, MNRAS, 471, 5018

Ferrara, A., Vallini, L., Pallottini, A., et al. 2019, MNRAS, 489, 1

Fudamoto, Y., Oesch, P. A., Faisst, A., et al. 2020, A\&A, 643, A4

Fujimoto, S., Silverman, J. D., Bethermin, M., et al. 2020, ApJ, 900, 1

Ginolfi, M., Jones, G. C., Béthermin, M., et al. 2020, A\&A, 633, A90

Glover, S. C. O., \& Smith, R. J. 2016, MNRAS, 462, 3011

Graciá-Carpio, J., Sturm, E., Hailey-Dunsheath, S., et al. 2011, ApJ, 728, L7

Gullberg, B., De Breuck, C., Vieira, J. D., et al. 2015, MNRAS, 449, 2883

Harikane, Y., Ouchi, M., Shibuya, T., et al. 2018, ApJ, 859, 84

Harikane, Y., Ouchi, M., Inoue, A. K., et al. 2020, ApJ, 896, 93

Hashimoto, T., Laporte, N., Mawatari, K., et al. 2018, Nature, 557, 392

Herrera-Camus, R., Bolatto, A. D., Wolfire, M. G., et al. 2015, ApJ, 800, 1

Hirashita, H., Burgarella, D., \& Bouwens, R. J. 2017, MNRAS, 472, 4587

Hollenbach, D. J., \& Tielens, A. G. G. M. 1999, Rev. Mod. Phys., 71, 173

Inoue, A. K., Tamura, Y., Matsuo, H., et al. 2016, Science, 352, 1559

Kelly, B. C. 2007, ApJ, 665, 1489

Kennicutt, R. C., Jr. 1998, ARA\&A, 36, 189

Knudsen, K. K., Richard, J., Kneib, J.-P., et al. 2016, MNRAS, 462, L6

Kohandel, M., Pallottini, A., Ferrara, A., et al. 2019, MNRAS, 487, 3007

Lagache, G., Cousin, M., \& Chatzikos, M. 2018, A\&A, 609, A130

Laporte, N., Ellis, R. S., Boone, F., et al. 2017, ApJ, 837, L21

Laporte, N., Katz, H., Ellis, R. S., et al. 2019, MNRAS, 487, L81

Le Fèvre, O., Béthermin, M., Faisst, A., et al. 2020, A\&A, 643, A1

Leung, T. K. D., Olsen, K. P., Somerville, R. S., et al. 2020, ApJ, submitted

[arXiv:2004.11912]

Madau, P., \& Dickinson, M. 2014, ARA\&A, 52, 415

Magdis, G. E., Rigopoulou, D., Hopwood, R., et al. 2014, ApJ, 796, 63

Maiolino, R., Carniani, S., Fontana, A., et al. 2015, MNRAS, 452, 54

Malhotra, S., Kaufman, M. J., Hollenbach, D., et al. 2001, ApJ, 561, 766

Marrone, D. P., Spilker, J. S., Hayward, C. C., et al. 2018, Nature, 553, 51

Matthee, J., Sobral, D., Boogaard, L. A., et al. 2019, ApJ, 881, 124

Murphy, E. J., Condon, J. J., Schinnerer, E., et al. 2011, ApJ, 737, 67 
Nordon, R., \& Sternberg, A. 2016, MNRAS, 462, 2804

Ota, K., Walter, F., Ohta, K., et al. 2014, ApJ, 792, 34

Ouchi, M., Ellis, R., Ono, Y., et al. 2013, ApJ, 778, 102

Pentericci, L., Carniani, S., Castellano, M., et al. 2016, ApJ, 829, L11

Pineda, J. L., Langer, W. D., \& Goldsmith, P. F. 2014, A\&A, 570, A12

Popping, G., Narayanan, D., Somerville, R. S., Faisst, A. L., \& Krumholz, M. R. 2019, MNRAS, 482, 4906

Riechers, D. A., Carilli, C. L., Capak, P. L., et al. 2014, ApJ, 796, 84

Romano, M., Cassata, P., Morselli, L., et al. 2020, MNRAS, 496, 875

Schaerer, D., de Barros, S., \& Sklias, P. 2013, A\&A, 549, A4

Schreiber, C., Elbaz, D., Pannella, M., et al. 2018, A\&A, 609, A30

Tamura, Y., Mawatari, K., Hashimoto, T., et al. 2019, ApJ, 874, 27

Vallini, L., Gallerani, S., Ferrara, A., Pallottini, A., \& Yue, B. 2015, ApJ, 813 36

Willott, C. J., Carilli, C. L., Wagg, J., \& Wang, R. 2015, ApJ, 807, 180

Wolfire, M. G., Hollenbach, D., McKee, C. F., Tielens, A. G. G. M., \& Bakes, E. L. O. 1995, ApJ, 443, 152

Wuyts, S., Förster Schreiber, N. M., Lutz, D., et al. 2011, ApJ, 738, 106

Zanella, A., Daddi, E., Magdis, G., et al. 2018, MNRAS, 481, 1976

1 Observatoire de Genève, Université de Genève, 51 Ch. des Maillettes, 1290 Versoix, Switzerland e-mail: daniel.schaerer@unige.ch

2 CNRS, IRAP, 14 avenue E. Belin, 31400 Toulouse, France

3 Aix Marseille Université, CNRS, CNES, LAM (Laboratoire d'Astrophysique de Marseille), 13013 Marseille, France

${ }^{4}$ IPAC, California Institute of Technology, 1200 East California Boulevard, Pasadena, CA 91125, USA

5 Cosmic Dawn Center (DAWN), Copenhagen, Denmark

${ }^{6}$ Niels Bohr Institute, University of Copenhagen, Lyngbyvej 2, 2100 Copenhagen, Denmark

7 Dipartimento di Fisica e Astronomia, Università di Padova, Vicolo dell'Osservatorio, 3, 35122 Padova, Italy

8 INAF, Osservatorio Astronomico di Padova, Vicolo dell'Osservatorio 5, 35122 Padova, Italy

9 Kavli Institute for the Physics and Mathematics of the Universe (Kavli IPMU, WPI), The University of Tokyo, 277-8583 Kashiwa, Japan

10 Department of Astronomy, School of Science, The University of Tokyo, 7-3-1 Hongo, Bunkyo, Tokyo 113-0033, Japan

11 The Caltech Optical Observatories, California Institute of Technology, Pasadena, CA 91125, USA

12 Cavendish Laboratory, University of Cambridge, 19 J. J. Thomson Ave., Cambridge CB3 OHE, UK

13 Kavli Institute for Cosmology, University of Cambridge, Madingley Road, Cambridge CB3 OHA, UK
${ }^{14}$ Instituto de Investigacion Multidisciplinar en Ciencia y Tecnologia, Universidad de La Serena, Raul Bitran 1305, La Serena, Chile

15 Departamento de Astronomia, Universidad de La Serena, Av. Juan Cisternas 1200 Norte, La Serena, Chile

16 INAF - Osservatorio di Astrofisica e Scienza dello Spazio di Bologna, Via Gobetti 93/3, 40129 Bologna, Italy

17 Centro de Astronomia (CITEVA), Universidad de Antofagasta, Avenida Angamos 601, Antofagasta, Chile

18 University of Bologna, Department of Physics and Astronomy (DIFA), Via Gobetti 93/2, 40129 Bologna, Italy

19 INAF - Osservatorio Astrofisico di Arcetri, Largo E. Fermi 5, 50125 Firenze, Italy

20 Astronomy Department, University of Massachusetts, Amherst, MA 01003, USA

21 Space Telescope Science Institute, 3700 San Martin Drive, Baltimore, MD 21218, USA

22 Instituto de Física y Astronomía, Universidad de Valparaíso, Avda. Gran Bretaña 1111, Valparaíso, Chile

23 Department of Physics, University of California, Davis, One Shields Ave., Davis, CA 95616, USA

24 Department of Astronomy, University of Florida, 211 Bryant Space Sciences Center, Gainesville, FL 32611, USA

25 University of Florida Informatics Institute, 432 Newell Drive, CISE Bldg E251, Gainesville, FL 32611, USA

26 Department of Astronomy, Cornell University, Space Sciences Building, Ithaca, NY 14853, USA

27 Max-Planck Institut für Astronomie, Königstuhl 17, 69117 Heidelberg, Germany

28 Leiden Observatory, Leiden University, PO Box 9500, 2300 RA Leiden, The Netherlands

\section{Appendix A: Fits for the [C ${ }_{\|]}-S F R$ relation at high redshift}

The ALPINE dataset and the data for $z>6$ galaxies from the literature were fitted using a Bayesian fit including censored data following the method of Kelly (2007), which is implemented in the linmix python package. In Table A.1, we list the resulting fit coefficients of the linear fits of the form $\log \left(L([\mathrm{CII}]) / L_{\odot}\right)=$ $a+b \times \log \left(\mathrm{SFR}_{\mathrm{tot}} / M_{\odot} \mathrm{yr}^{-1}\right)$ and their uncertainties obtained for different combinations of datasets, assumptions on SFR(tot), and adopted [C II] upper limits. Not all combinations are shown and discussed in the text; those shown in figures are indicated in the last column in the table.

Table A.1. Fit coefficients from Bayesian fits including censored data: $(a, b)=$ (offset, slope) and their uncertainties (standard deviation).

\begin{tabular}{llllllll}
\hline \hline Dataset & SFR & {$[\mathrm{C}$ II] limits } & Offset & Std (offset) & Slope & Std (slope) & Fig. \\
\hline ALPINE & UV+IR & $3-\sigma$ limits & 7.03 & 0.17 & 1.00 & 0.12 & 2 \\
ALPINE & UV+IR & $6-\sigma$ & 7.37 & 0.14 & 0.83 & 0.10 & \\
ALPINE & SED & $3-\sigma$ & 7.09 & 0.21 & 0.84 & 0.13 & 3 right \\
ALPINE & SED & $6-\sigma$ & 7.43 & 0.17 & 0.70 & 0.10 & \\
ALPINE & UV+IRX & $3-\sigma$ & 6.61 & 0.20 & 1.17 & 0.12 & 4 \\
ALPINE & UV+IRX & $6-\sigma$ & 7.05 & 0.15 & 0.96 & 0.09 & 6 \\
ALPINE $+z>6$ & UV+IRX & $3-\sigma$ & 6.43 & 0.16 & 1.28 & 0.10 & 7 \\
ALPINE $+z>6$ & UV+(IRX for ALPINE only) & $3-\sigma$ & 6.51 & 0.15 & 1.24 & 0.10 & \\
ALPINE $+z>6$ & UV+IRX & $6-\sigma$ & 6.66 & 0.14 & 1.17 & 0.09 & \\
\hline
\end{tabular}

Notes. Column 1 indicates the dataset used, Col. 2 the total SFR used, Col. 3 the [C II] limits. Column 8 indicates the figure number showing the corresponding data and fit in some cases. 